\title{
Le vaccin contre le virus du papillome humain : risques et réalités
}

Publié sur www.cmaj.ca le $1^{\mathrm{er}}$ septembre 2008.

A u Canada l'an dernier, les programmes scolaires de vaccination contre le virus du papillome humain (VPH) ont suscité de vives préoccupations. Ce fut particulièrement le cas en Ontario, où l'acceptation du vaccin a atteint à peine $50 \%$, écart important par rapport aux $80 \%$ et plus atteints en Nouvelle-Écosse et en Australie. En guise de comparaison, le vaccin contre l'hépatite B est accepté à plus de $90 \%$ dans les programmes scolaires au Canada. Pourquoi donc le vaccin contre le VPH est-il moins accepté qu'on l'espérait étant donné que le programme vise à prévenir la cancer du col, maladie qui peut être mortelle?

Cassels ${ }^{1}$ a affirmé que le débat sur le vaccin confronte ceux qui veulent agir pour éviter d'éventuels décès attribuables au cancer du col à ceux qui se demandent ce qui presse tant et pensent aux inconnus qui restent encore à cerner, notamment les coûts, l'efficacité et l'innocuité. Le débat a été utile pour ceux qui l'ont suivi attentivement, mais certains parents et des bénéficiaires éventuelles du vaccin qui ont décidé de ne pas participer au programme n'en ont peut-être entendu que des bribes.

Il y a des leçons à tirer en l'occurrence pour les pays d'Europe et les autres provinces canadiennes qui s'apprêtent à lancer des programmes de vaccination contre le VPH. Pour donner confiance envers tout programme de vaccination, il faut prévoir les préoccupations des parents et des bénéficiaires éventuelles du vaccin et y répondre proactivement; il faut particulièrement répondre à ceux qui se demandent ce qui presse tant. Il est crucial d'insister sur les données démontrant l'innocuité du vaccin, ses avantages et les risques de maladie.

Dans ce numéro, Brotherton et ses collaborateurs ${ }^{2}$ présentent des preuves convaincantes de l'innocuité remarquable du vaccin contre le VPH. En Australie, de très rares cas d'anaphylaxie (2,6 cas pour 100000 doses) ont constitué les seuls événements indésirables graves signalés après un suivi attentif de plus de 260000 doses de vaccin. Il n'y a eu aucun cas de choc anaphylactique. Toutes les jeunes filles se sont rétablies complètement, habituellement rapidement après avoir reçu de l'épinéphrine. Dans un commentaire connexe, Halsey ${ }^{3}$ souligne encore davantage la rareté de l'anaphylaxie et autres effets indésirables graves à la suite de l'administration de millions de doses du vaccin. Ces données devraient rassurer les parents et les bénéficiaires éventuelles qui ont demandé davantage de preuves de l'innocuité du vaccin.

L'étude réalisée par Brotherton et ses collaborateurs donne aux milieux canadiens de la santé publique une excellente occasion de relancer les discussions publiques sur l'innocuité du vaccin contre le VPH, les précautions prises pour atténuer les risques en cas d'anaphylaxie et le soin pris pour surveiller les événements indésirables après une vaccination ${ }^{4}$. Ces programmes scolaires de vaccination obligent les fournisseurs du vaccin à avoir la formation et le matériel nécessaires pour traiter les cas d'anaphylaxie et observer les bénéficiaires pendant 15 minutes après la vaccination. Il faut satisfaire à ces exigences fondamentales pour fournir le vaccin dans n'importe quel contexte ${ }^{4}$

Les campagnes de santé publique doivent tenir compte du fait que les parents et les bénéficiaires éventuelles du vaccin s'arrêtent plus volontiers aux préoccupations immédiates que lointaines en matière de santé. Pourtant, le cancer du col, risque qui peut sembler lointain pour une adolescente, frappera quelque 1300 femmes cette année au Canada et 380 en mourront ${ }^{5}$. Ce risque persiste même après des décennies de campagnes de santé publique sur l'importance du dépistage régulier.
Retarder la vaccination jusqu'à ce qu'une jeune femme soit sexuellement active semblerait constituer une solution crédible, mais ce n'est pas le cas. La première relation sexuelle peut causer une infection par le VPH et la moitié des jeunes femmes du Canada sont sexuellement actives à 16 ans. Le facteur de risque lié aux comportements des adolescentes sont des prédicteurs insuffisants d'infections futures pour le VPH de type vaccinal ${ }^{6}$.

Idéalement, des taux élevés de vaccination contre le VPH réduiraient le risque de cancer du col tant chez les femmes non vaccinées que chez celles qui le sont à cause de l'immunité collective. Or, il y a actuellement peu de chance que cela se produise parce qu'il faudrait vacciner autant les hommes que les femmes. Le Canada et d'autres pays industrialisés (sauf l'Australie) ont approuvé la vaccination pour les femmes seulement jusqu'ici parce qu'on n'a pas terminé les études sur les hommes. C'est pourquoi seules les femmes immunisées seront protégées pour le moment.

En publiant ces données sur l'innocuité du vaccin, le JAMC continue de participer au débat sur la vaccination contre le VPH. Les praticiens de la santé publique et autres doivent maintenant écouter attentivement les préoccupations du public et y répondre. Afin de respecter le droit de chaque femme de décider elle-même de se faire vacciner ou non contre le VPH, il faut notamment assurer que cette décision repose sur une opinion équilibrée et factuelle des risques concurrents, plutôt que sur l'ignorance ou sur des peurs irrationnelles.

Noni MacDonald MD MSc

Rédactrice de la section Santé publique

Matthew B. Stanbrook MD PhD

Rédacteur adjoint, Sciences

Paul C. Hébert MD MHSc

Rédacteur en chef, JAMC

Avec l'équipe de rédaction de l'éditorial (Rajendra Kale MD, Barbara Sibbald BJ, Ken Flegel MDCM MSc

et Amir Attaran LLB DPhil)

Intérêts concurrents : Voir www.cmaj.ca/misc/edboard.shtml.

Traduit par le Service de traduction de l'AMC.

La version française de l'éditorial a été quelque peu abrégée en raison de contraintes d'espace.

\section{RÉFÉRENCES}

1. Cassels AK. Vaccination against human papilloma virus [lettre]. CMAJ 2007;177:1526.

2. Brotherton JML, Gold MS, Kemp AS, et al. Anaphylaxis following quadrivalent human papillomavirus vaccination. CMAJ 2008;179:525-33.

3. Halsey NA. The human papillomavirus vaccine and risk of anaphylaxis. CMAJ 2008;179:509-10.

4. Agence de la santé publique du Canada. Guide canadien d'immunisation. 7e éd. Ottawa (Ont.) : l'Agence; 2006. Disponible : www.phac-aspc.gc.ca/publicat/cig-gci/ index-fra.php (consulté le 5 août 2008).

5. Société canadienne du cancer. Statistiques canadiennes sur le cancer. Toronto (Ont.) : la Société; 2008. Disponible : www.cancer.ca/ccs/internet/standard /0,3182,3172_14279_langId-fr,00.html (consulté le 5 août 2008).

6. Dempsey AF, Gebremariam A, Koutsky L, et al. Behavior in early adolescence and risk of human papillomavirus infection as a young adult: results from a population-based study. Pediatrics 2008;122:1-7. 